Likewise the beautiful theorem which asserts that two permutable substitutions must have a common pole is omitted.

The author follows Jordan, Klein, and Burnside in writing the accented, i. e., the new, variables on the left, and writes the factors of a product in such order that multiplication of substitutions is effected through columns-by-rows multiplication of the matrices.

In spite of the omission of some things that the reviewer would have liked to see included, the book is a noteworthy contribution to a subject that is of increasing importance to students of mathematics. It is well written, though the condensed notation makes it rather difficult reading for the beginner in the subject.

ERNest B. Skinner.

\title{
MATHEMATICS AT AN ITALIAN TECHNICAL SCHOOL.
}

ThE regular courses in the Italian technical schools are five years long, most of the work being prescribed. The schedule is not exactly the same in all of them, yet the general scheme followed can be understood by following the plan of instruction in any one of them. For this purpose we choose that at Milan (Reale Istituto Tecnico Superiore di Milano), which includes a two-year course in mathematics, physics, and chemistry and also in Italian and two foreign languages as preparatory to the regular three-year courses in mechanical, electric, civil, and mining engineering, architecture, industrial, physical, and electric chemistry, and a four-year normal course. Candidates for admission must have a diploma from a recognized Italian secondary school or equivalent credentials. In mathematics they must be familiar with plane and solid geometry, plane trigonometry, algebra including determinants, theory of equations and graphical processes, and the elements of projective geometry.

The following outline applies to all the students except those in architecture, who have briefer courses in all the subjects mentioned.

During both terms of the first year there are three weekly lectures and one hour of exercises in plane and solid analytic 
geometry (Professor A. Jorini); four lectures and three hours of exercises in mathematical analysis (Professor U. Cisotti); three hours in physics, six in drawing, four (no laboratory) in chemistry, one in Italian literature, and two each in English and German. The first term's work is very similar to that given under the same heading in our American institutions, the main difference being that much less time is given to exercises. But while the internal structure in analytic geometry is similar, there is a marked difference in the work in mathematics as a whole, for during this same term the student also takes a much more comprehensive course in the calculus. It begins with a review of determinants, systems of linear equations, complex numbers, and the idea of functionality. Derivatives of algebraic functions are introduced as soon as the student has completed the geometry of the straight line, and it is at once followed by a generous treatment of maxima and minima. In the treatment of series general questions of convergence are studied, the theorems of Taylor and Maclaurin derived, and the remainder employed. The next chapter is that on geometric applications, including tangents, inflexions, curvature, evolutes, and singular points.

In analytics, the entire second term is devoted to space; the instruction includes an extensive discussion of quadric surfaces, making free use of polar properties. In analysis, we begin with integration as a formal process, then as summation, followed by the usual elementary geometric applications. Then go back to functions of two or more variables, implicit functions and some geometric applications of partial derivatives. Finally, a short survey of elliptic integrals, integration by series, and a glimpse at differentiation and integration under the sign of integration.

In the second year analysis is taught six hours per week during the first semester (Professor U. Cisotti), projective and descriptive geometry is taught throughout the year, three lectures and six hours of drawing per week (Professor C. Capelli), and rational mechanics both terms, four lectures and one hour of exercises per week (Professor B. Caldonazzo). The hours in physics, chemistry, and languages are about the same as for the first year. The number of actual hours per week required of each student is 30 the first year, 38 the second. In analysis, the topics considered are space curves, multiple 
integration, differential equations, approximate solutions, Fourier series, functions of a complex variable, conformal representation, and an introduction to vector analysis.

In projective geometry the work is taken up in the sequence and about the extent treated in Cremona's book* followed by reciprocal radii and general quadratic inversion. In descriptive geometry, the course given is about the same as is found in our better institutions, with the addition of further work on axonometry, central perspective, ruled surfaces of orders three and four, and brilliant points and lines.

The course in mechanics begins with vector analysis followed by statics, including center of gravity, then by kinematics of a point and of a rigid body, precession and relative motion, ellipsoid of inertia. After about two months dynamics is introduced, mass, weight, force defined, impulses, kinetic energy, free and restrained vibrations, resonance, gravitation, central motion, Kepler's law, and the centrifugal pendulum being studied. In the second term the topics considered are d'Alembert's principle, potential energy, examples, including loop the loop, stability of equilibrium, dynamics of a rigid body, compound pendulum, Euler's equations, the gyroscope, and various applications. Finally, dynamics of continuous systems, hydrostatics and hydrodynamics, equations of Laplace and Poisson, lines and tubes of flux, gravitational field, potential of spherical strata, etc.

This completes the regular work in mathematics. During the third year courses differ for students in the various branches, but all take the thermodynamics (three hours), and applied mechanics, nine hours per week.

If we include rational mechanics and projective geometry in pure mathematics, but exclude descriptive geometry, thermodynamics and applied mechanics, we see that the Italian technical school student has thirty-six hours per week for one term of pure mathematics, and has had preliminary work at least equal to that required of our most exacting schools. Moreover, a more advanced course in analysis is offered as an elective by Professor G. Vivanti, to be taken in the fourth or fifth year, and similar choices are possible in several branches of applied mathematics.

VIRGIL SNYDER.

* Cremona was professor of geometry at the Milan technical school when he wrote his well-known treatise on projective geometry. 